\title{
Reduction of blood nitric oxide levels is associated with clinical improvement of the chronic pelvic pain related to endometriosis
}

\author{
M.G. Rocha ${ }^{1}$, V.A. Gomes ${ }^{2}$, J.E. Tanus-Santos ${ }^{3}$, J.C. Rosa-e-Silva ${ }^{4}$, F.J. Candido-dos-Reis ${ }^{4}$, \\ A.A. Nogueira ${ }^{4}$ and O.B. Poli-Neto ${ }^{4}$ \\ ${ }^{1}$ Faculdade de Medicina, Universidade de Fortaleza, Fortaleza, CE, Brasil \\ ${ }^{2}$ Departamento de Farmacologia, Hospital Universitário, Faculdade de Ciências Médicas, Universidade Estadual de Campinas, \\ Campinas, SP, Brasil \\ ${ }^{3}$ Departamento de Farmacologia, Faculdade de Medicina de Ribeirão Preto, Universidade de São Paulo, \\ Ribeirão Preto, SP, Brasil \\ ${ }^{4}$ Departamento de Ginecologia e Obstetrícia, Hospital das Clínicas, Faculdade de Medicina de Ribeirão Preto, \\ Universidade de São Paulo, Ribeirão Preto, SP, Brasil
}

\begin{abstract}
The objective of this prospective study was to determine the plasma levels of nitric oxide (NO) in women with chronic pelvic pain secondary to endometriosis $(n=24)$ and abdominal myofascial pain syndrome $(n=16)$. NO levels were measured in plasma collected before and 1 month after treatment. Pretreatment NO levels $(\mu \mathrm{M})$ were lower in healthy volunteers $(47.0 \pm 12.7)$ than in women with myofascial pain $(64.2 \pm 5.0, P=0.01)$ or endometriosis $(99.5 \pm 12.9, P<0.0001)$. After treatment, plasma NO levels were reduced only in the endometriosis group $(99.5 \pm 12.9$ vs $61.6 \pm 5.9, P=0.002)$. A correlation between reduction of pain intensity and reduction of $\mathrm{NO}$ level was observed in the endometriosis group [correlation $=0.67$ $(95 \% \mathrm{Cl}=0.35$ to 0.85$), \mathrm{P}<0.0001]$. Reduction of $\mathrm{NO}$ levels was associated with an increase of pain threshold in this group [correlation $=-0.53(-0.78$ to -0.14$), \mathrm{P}<0.0001$ ]. NO levels appeared elevated in women with chronic pelvic pain diagnosed as secondary to endometriosis, and were directly associated with reduction in pain intensity and increase in pain threshold after treatment. Further studies are needed to investigate the role of NO in the pathophysiology of pain in women with endometriosis and its eventual association with central sensitization.
\end{abstract}

Key words: Chronic pelvic pain; Endometriosis; Abdominal myofascial pain syndrome; Nitric oxide; Inflammation; Central sensitization

\section{Introduction}

Chronic pelvic pain (CPP) is a highly prevalent (2 to $25 \%$ ) clinical problem among women (1). It is described as continuous or intermittent pain in the anatomic pelvis (anterior abdominal wall at or below the umbilicus) that lasts at least 6 months, is not exclusively related to menstruation or sexual intercourse, and is sufficiently severe to cause functional disability or to lead to medical care (2). Its etiology is often unknown but may result from a complex interaction between the gastrointestinal, urinary, gynecologic, musculoskeletal, neurologic, and endocrine systems. It is also influenced by psychological and sociocultural factors. Among women seen at gynecology outpatient clinics, one of the diseases most often associated with CPP is endometriosis.
Abdominal myofascial pain syndrome, characterized by hyperirritable trigger points is another frequent underdiagnosed condition causing CPP, especially in countries where the prevalence of cesarean section and other abdominal surgeries is common (3).

Researchers, physicians, gynecologists and patients believe that CPP can be caused by the endometriosis lesions (4), but determining how the lesions cause CPP has proved difficult. Various pathophysiological mechanisms have been proposed to explain the development of myofascial pain syndromes, but little is known about the cause of this condition. The chronic inflammatory response in endometriosis has been identified as an important factor

Correspondence: O.B. Poli-Neto: <polineto@fmrp.usp.br>. 
in the pathophysiology of the disease (5). Similarly, elevated levels of inflammatory mediators have been reported in sites close to or far from active myofascial trigger points (6). Some studies indicate a possible role for nitric oxide (NO) in the pathogenesis of endometriosis (7). It is now well appreciated that NO can act as a mediator and regulator of the inflammatory response (8) and of the modulation of nociception at both the peripheral and the central level (9). Studies on animals have demonstrated that $\mathrm{NO}$ enhances the sensitivity of peripheral nociceptors (10) and can contribute to hyperalgesia secondary to central sensitization (11). Most studies have shown pronociceptive properties of $\mathrm{NO}$ in the spinal cord. Nevertheless, NO has a dual role in pain pathways. There is also evidence of its antinociceptive and analgesic effect, particularly when present at low concentrations within the spinal cord (12). In addition to this direct analgesic effect, it may also mediate the peripheral and central antinociceptive effect of other analgesic compounds (e.g., opioids and NSAIDs) (9). In humans, intracutaneous NO injection can evoke pain (13), and increased NO levels have been detected in the plasma of patients with chronic pain (14), temporomandibular joint disorders (15), chronic orofacial pain (16), and bladder pain syndrome/interstitial cystitis (17). Although it has been observed that greater amounts of $\mathrm{NO}$ are present in the endometrial tissues of women with endometriosis (7), no studies have investigated plasma $\mathrm{NO}$ levels in women with CPP secondary to endometriosis, nor has it been determined whether there are differences between women with CPP and those with abdominal myofascial pain syndrome. Thus, the objective of the present study was to determine plasma NO levels in women with CPP secondary to those conditions and to explore their association with pain intensity and pain threshold.

\section{Material and Methods}

\section{Study population}

This prospective study was conducted in women of reproductive age without infertility and with regular menstrual cycles. The Ethics Committee of the Hospital das Clínicas, Faculdade de Medicina de Ribeirão Preto, Universidade de São Paulo approved the study (protocol \#3502/ 2007, national registry 0112.0.004.000-07). The study samples were obtained when all inclusion criteria were satisfied and written informed consent was obtained. The research subjects were women with CPP and treated at the Gynecologic Endoscopy and Chronic Pelvic Pain Center of the same institution. Data from healthy control women during routine gynecological follow-up were matched with the study subjects by date of blood collection, age and parity, and were obtained from a database of primary health care unit records maintained by the university.

We selected 25 healthy subjects and 40 women with CPP without previous treatment. Women with CPP were divided into 2 groups. Group 1 included 24 women diagnosed with endometriosis confirmed by histology and classified during laparoscopy according to the American Society for Reproductive Medicine (18) as moderate $(n=14)$ or severe $(n=10)$. Women with intestinal, bladder, or rectovaginal septum invasion by endometriosis, as well as women with minimal or mild endometriosis because of extensive surgery or lack of histologic confirmation, respectively, were excluded from the study. Group 2 included 16 women diagnosed exclusively with abdominal myofascial pain syndrome (19). The study thus included a healthy control group, an endometriosis group, and a myofascial group. Subjects with a history of inflammation including fever, a history of acute diseases such as flu; chronic diseases such as rheumatoid arthritis, lupus, asthma, and allergic reactions; hypertension, smoking habit, alcoholism, using hormonal contraceptives, or without an imputable cause for CPP, were also excluded (Figure 1).

\section{Procedures}

Women with endometriosis underwent laparoscopy under general anesthesia and with pneumoperitoneum obtained using carbon dioxide. Judicious inspection of the abdominopelvic cavity was performed before any other procedures were carried out. Endometriotic lesions were classified according to the American Society of Reproductive Medicine classification system (18). Electrosurgical ablation and/or excision of superficial lesions that were identified, removal of deep lesions, and/or cystectomy for endometriomas were performed. All women underwent conservative surgery, preserving the ovaries.

The diagnosis of abdominal myofascial pain syndrome was clinically confirmed by Simons' criteria (19). All women with this syndrome were examined by abdominal wall ultrasound in order to exclude any other local conditions such as hernias or abdominal wall cystic endometriosis. Women with signs of ilioinguinal/iliohypogastric/genitofemoral neuropathies were excluded. No women in this group presented with significant dysmenorrhea. As specified in our service protocol, all women in the myofascial group received injections of $2 \mathrm{~mL} 0.5 \%$ lidocaine (20), without a vasoconstrictor, directly in and perpendicular to the trigger point at 5 weekly sessions.

\section{Pain measurement}

Pain was quantified using a visual analogue scale (VAS) before and after treatment; the McGill pain questionnaire was applied only before treatment (21). We considered the reduction of pain to be clinically relevant when subjects showed a $50 \%$ reduction in the VAS, or at least a $20 \mathrm{~mm}$ change with significant improvement reported in daily activities (22).

The pressure pain threshold (i.e., the minimal pressure that induced pain) was assessed with an Instrutherm DD500 pressure algometer with digital traction and compression and a measuring capacity of $5 \mathrm{~kg}$ (Digital Dynamometer, Model: DD200, Serial No. 08030300338819 , Instrutherm Measuring Instruments Ltd., Brazil). The apparatus consisted of 


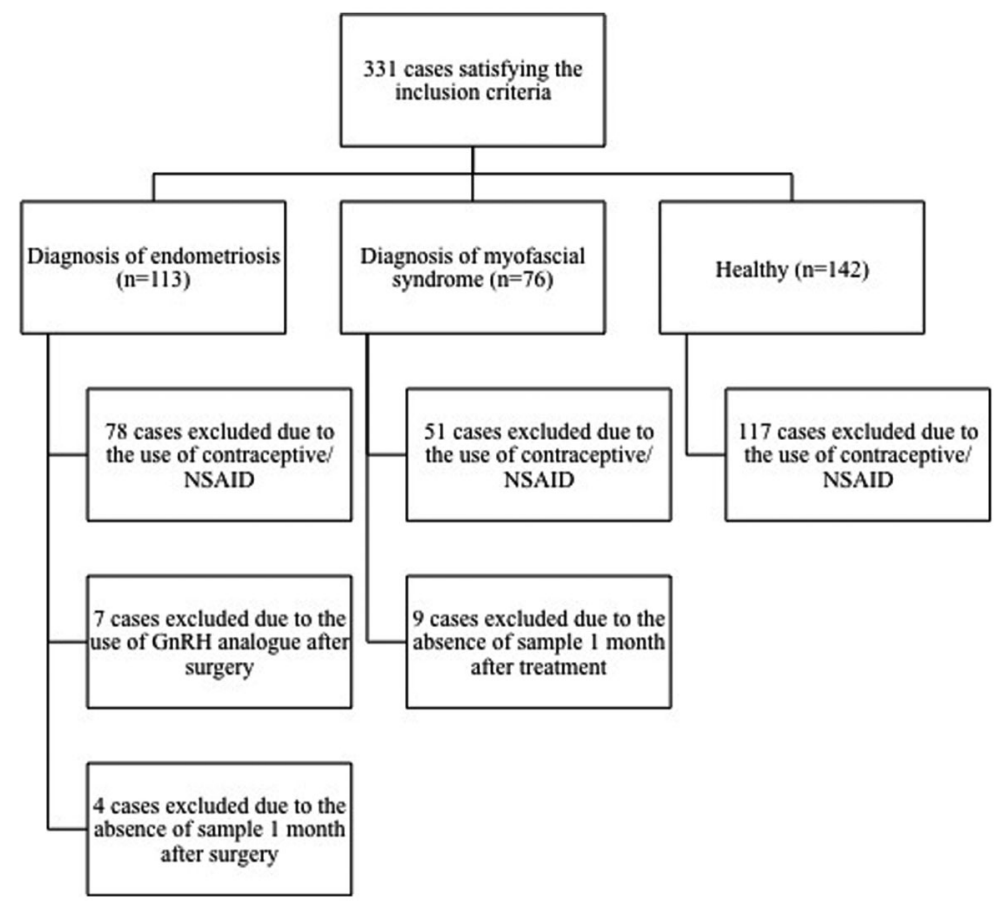

Figure 1. Flow diagram of the recruitment of subjects and samples.

a 1-cm diameter rubber disk attached to the plunger of a pressure (force) gauge. The dial of the gauge was calibrated in $\mathrm{kg} / \mathrm{cm}^{2}$. We routinely measured the threshold at a single point on the thenar region of the nondominant hand. Three consecutive measurements were taken with an interval of $20 \mathrm{~min}$ and the means were used in the analysis. The Hospital Anxiety and Depression Scale $(23,24)$ was also used as a psychometric instrument.

\section{NO measurement}

Blood samples were obtained from women in the follicular phase of the menstrual cycle (fifth to ninth day) between 7:00 and 9:00 am (25). The first sample was collected immediately before treatment and the second at 4 weeks after treatment. Subjects whose data were included in the database were routinely contacted by phone 3 to 4 days before the collection and advised about diet (to avoid foods such as meats in excessive amounts, coffee, teas, chocolates, spices, pepper, salt, among others) and drug intake (to avoid analgesics and nonsteroidal anti-inflammatory drugs). Plasma samples were stored at $-70^{\circ} \mathrm{C}$ until nitrate was assayed by the Griess colorimetric reaction (26).

Nitrite $\left(\mathrm{NO}_{2}\right)$ plus nitrate $\left(\mathrm{NO}_{3}\right)$ levels were determined in plasma samples by enzymatically reducing nitrate with bacterial nitrate reductase. Briefly, duplicate plasma samples $(40 \mu \mathrm{L})$ were incubated overnight at $37^{\circ} \mathrm{C}$ with an equal volume of reductase buffer $(0.5 \mathrm{M}$ potassium phosphate, $\mathrm{pH} 7.5$, containing NADPH and nitrate reductase). The total amount of nitrate was then determined by the colorimetric Griess reaction. Briefly, samples were incubated with $80 \mu \mathrm{L}$ freshly prepared Griess reagent (2\% sulphanylamide in $5 \%$ phosphoric acid and $0.2 \%$ naphthylethylenediamine dihydrochloride). After $15 \mathrm{~min}$ of color development at room temperature, the sample absorbance at $540 \mathrm{~nm}$ was compared with values on a standard nitrate curve. The results are reported as $\mu \mathrm{M} \mathrm{NO}_{3}+\mathrm{NO}_{2}$.

\section{Statistical analysis}

Comparisons of $\mathrm{NO}$ levels between groups were performed using the nonparametric Wilcoxon test for each pair. The Wilcoxon signed rank test was used to compare NO between pre- and post-treatment samples. Correlations between NO, VAS, and pain threshold were evaluated by the multivariate restricted maximum likelihood method. For that analysis, we defined pain intensity variation ( $\triangle \mathrm{VAS}$ ) as (post-treatment VAS-pretreatment VAS)/pretreatment VAS) $\mathrm{NO}$ level variation ( $\triangle \mathrm{NO}$ ) was (post-treatment NOpretreatment NO)/pretreatment NO. Pain threshold variation ( $\Delta$ threshold) was (post-treatment threshold-pretreatment threshold)/pretreatment threshold). A negative sign was used to indicate a reduction of measured value. The statistical tests and plots were performed using JMP 10 statistical software for Mac (SAS Institute, USA) with the level of significance set at $\mathrm{P}<0.05$.

\section{Results}

The characteristics of the study participants are presented in Table 1. There was a clinically relevant reduction of pain in $91.7 \%(n=22 / 24)$ of women with endometriosis and $75.0 \%(n=12 / 16)$ of women with abdominal myofascial pain syndrome. Pretreatment NO levels $(\mu \mathrm{M})$ were lower in 
Table 1. Characteristics of the subjects included in the study.

\begin{tabular}{|c|c|c|c|c|}
\hline Parameters & Healthy $(n=25)$ & Endometriosis $(n=24)$ & Myofascial $(n=16)$ & $\mathrm{P}$ \\
\hline Age (years) & $35.4 \pm 6.7$ & $32.9 \pm 9.0$ & $32.7 \pm 7.8$ & 0.37 \\
\hline Parity (median, range) & $1(0-5)$ & $1.5(0-11)$ & $1(0-4)$ & 0.30 \\
\hline $\mathrm{BMI}$ & $25.0 \pm 3.3$ & $25.6 \pm 5.9$ & $26.1 \pm 3.4$ & 0.66 \\
\hline HAD-anxiety & $6.4 \pm 4.1$ & $9.6 \pm 5.0$ & $10.1 \pm 6.9$ & 0.09 \\
\hline$>8(\mathrm{n}, \%)$ & $6(24.0)$ & $12(50.0)$ & $5(31.2)$ & 0.15 \\
\hline HAD-depression & $6.5 \pm 5.0$ & $8.3 \pm 4.6$ & $10.8 \pm 6.7$ & 0.13 \\
\hline$>9, \mathrm{n}(\%)$ & $7(28.0)$ & $8(33.3)$ & $5(31.2)$ & 0.92 \\
\hline Duration of pain, months & - & $37.2 \pm 31.7$ & $40.6 \pm 28.2$ & 0.54 \\
\hline 6 to 12 months, n (\%) & - & 7 (29.2) & $2(12.4)$ & \\
\hline 12 to 24 months, n (\%) & - & $4(16.7)$ & $3(18.8)$ & \\
\hline$>24$ months, $\mathrm{n}(\%)$ & - & $13(54.2)$ & $11(68.8)$ & \\
\hline VAS & - & $79.5 \pm 15.4$ & $85.9 \pm 11.0$ & 0.21 \\
\hline McGill & - & $33.3 \pm 14.8$ & $29.6 \pm 16.2$ & 0.51 \\
\hline Red blood cells & $4.6 \pm 0.3$ & $4.7 \pm 0.4$ & $4.5 \pm 0.3$ & 0.55 \\
\hline Hematocrit & $40.5 \pm 2.9$ & $41.4 \pm 3.8$ & $39.9 \pm 2.8$ & 0.30 \\
\hline Hemoglobin & $13.4 \pm 1.0$ & $13.7 \pm 1.3$ & $13.2 \pm 0.9$ & 0.30 \\
\hline White blood cells & $7.9 \pm 2.3$ & $7.4 \pm 3.0$ & $6.8 \pm 1,1$ & 0.20 \\
\hline Neutrophils & $56.3 \pm 11.0$ & $57.8 \pm 11.0$ & $58.9 \pm 8.4$ & 0.87 \\
\hline Lymphocytes & $33.0 \pm 9.2$ & $32.1 \pm 8.9$ & $32.3 \pm 7.7$ & 0.98 \\
\hline Eosinophils & $3.4 \pm 3.0$ & $2.6 \pm 2.1$ & $1.6 \pm 0.7$ & 0.03 \\
\hline Basophils & $0.6 \pm 0.3$ & $0.7 \pm 0.4$ & $0.8 \pm 0.5$ & 0.51 \\
\hline Monocytes & $6.5 \pm 3.1$ & $6.6 \pm 2.3$ & $6.4 \pm 1.8$ & 0.89 \\
\hline Platelets & $261.7 \pm 51.3$ & $269.5 \pm 60.8$ & $254.9 \pm 50.3$ & 0.97 \\
\hline
\end{tabular}

Data are reported as means \pm SD unless otherwise indicated. BMI: body mass index; HAD: Hospital Anxiety and Depression Scale; VAS: visual analogue scale, in millimeters; SD: standard deviation. The Wilcoxon and chi-squared tests were used for the statistical analyses.

healthy volunteers $(47.0 \pm 12.7)$ than in the myofascial $(64.2 \pm 5.0, \mathrm{P}=0.01)$ or endometriosis $(99.5 \pm 12.9, \mathrm{P}<$ $0.0001)$ groups. The latter group had higher pretreatment NO levels than the myofascial group $(P=0.005)$.

The pain thresholds $\left(\mathrm{kg} / \mathrm{cm}^{2}\right)$ of healthy women $(2.6 \pm$ 0.2 ) were significantly higher than those of women with endometriosis $(1.0 \pm 0.1, \mathrm{P}<0.0001)$ or myofascial syndrome $(1.9 \pm 0.2, P=0.007)$. This last group had higher pretreatment pain thresholds than the group of women with endometriosis $(P=0.0004)$.

There was a reduction of plasma NO levels after treatment in the endometriosis group $(99.5 \pm 12.9$ vs $61.6 \pm 5.9$, $\mathrm{P}=0.002$ ), but not in the myofascial group (64.2 $\pm 5.0 \mathrm{vs}$ $61.1 \pm 8.2 ; \mathrm{P}=0.70)$. No differences were observed in posttreatment $\mathrm{NO}$ levels between the study groups and controls even though the analysis had sufficient power to demonstrate it $(P=0.09)$. Table 2 shows post-treatment data regarding the VAS pain intensity, pain threshold and NO levels in the endometriosis and myofascial groups.

We observed a correlation between $\triangle \mathrm{VAS}$ and $\triangle \mathrm{NO}$ level in the endometriosis group [correlation $=0.67(95 \% \mathrm{Cl}$ : 0.35 to 0.85$), P<0.0001]$, but not in the myofascial group [correlation $=-0.64(-0.89$ to 0.10$), P=0.20$ ]. We also observed a correlation between $\Delta$ threshold and $\Delta \mathrm{NO}$ level in the endometriosis group [correlation $=-0.53(-0.78$ to $-0.14), \mathrm{P}<0.0001$ ] but not in the myofascial group [correlation $=-0.12(-0.65$ to 0.49$), P=0.88]$.

\section{Discussion}

The present study showed that women with CPP secondary to endometriosis had significantly elevated plasma NO levels compared with healthy controls and women with CPP secondary to abdominal myofascial pain syndrome. Clinical improvement of CCP after surgical treatment of endometriosis was associated with a reduction of plasma NO levels. However, these findings were not observed in women with myofascial syndrome. This suggests that ablation or excision of endometriosis may be responsible for reducing peripheral levels of NO. Indeed, injection of anesthetic into myofascial syndrome trigger points does not eliminate the injury and probably only modulates the afferents. Additionally, we observed that NO reduction in women with endometriosis was directly associated with reduction of pain threshold, suggesting that NO may be involved in the process of central sensitization. Interestingly, NO levels did not reach those seen in healthy women, even after appropriate treatment of their condition. It is possible that 
Table 2. Intensity of pain, pain threshold and nitric oxide levels after treatment for both the endometriosis and myofascial groups.

\begin{tabular}{lccccccc}
\hline & \multicolumn{2}{c}{ Endometriosis $(n=24)$} & & & \multicolumn{2}{c}{ Myofascial $(n=16)$} & $P$ \\
\cline { 2 - 3 } & Pre-treatment & Post-treatment & & & Pre-treatment & Post-treatment \\
\hline VAS (means \pm SD) & $79.5 \pm 15.4$ & $30.8 \pm 19.4$ & $<0.0001$ & $85.9 \pm 11.0$ & $26.1 \pm 5.7$ & $<0.001$ \\
Pain threshold (means \pm SE) & $1.0 \pm 0.1$ & $1.3 \pm 0.2$ & 0.21 & $1.9 \pm 0.2$ & $1.7 \pm 0.2$ & 0.98 \\
NO level (means \pm SE) & $99.5 \pm 12.9$ & $61.6 \pm 5.9$ & & 0.002 & $64.2 \pm 5.0$ & $61.1 \pm 8.2$ & 0.70 \\
\hline
\end{tabular}

NO: nitric oxide; VAS: visual analogue scale, in millimeters. The Wilcoxon signed rank test was used for the statistical analyses.

other, particularly central, pathophysiological events may be associated with the origin of $\mathrm{NO}$, but our study does not allow further evaluation.

Published studies have shown that NO may be involved in the pathophysiology of endometriosis. It has been observed that NO synthesis is increased in endometrial tissue from women with endometriosis (7), and changes have been observed in endothelial nitric oxide synthase (eNOS) expression in the endometrium of women with endometriosis $(27,28)$ that can be associated with specific gene polymorphisms of eNOS (29). However, eNOS has not been involved in pain processing (30). Furthermore, a significant increase in $\mathrm{NO}$ level was also detected in the peritoneal fluid of women with endometriosis (19), probably due to increased expression of inducible nitric oxide synthase (iNOS) in peritoneal macrophages (31).

Most previous studies have focused on women with infertility, and we did not find any that were specific to NO in women with CPP. The association between NO and pain is complex, but our results are similar to those reported by some other authors $(14,17)$. Under chronic pain conditions, we know that the activation of $\mathrm{N}$-methyl-D-aspartate (NMDA) receptors in the spinal cord can produce persistent enhancement of pain via production of $\mathrm{NO}$ and/or prostaglandins $(32,33)$. There is also evidence that NO may be directly involved in the process of central sensitization following a painful stimulus $(11,34)$. Our results $(9,12)$ suggest that this may be happening in women with CPP secondary to endometriosis, since there was a positive correlation between the reduction in plasma NO levels and pain thresholds measured at a distance from the primary site of pain. Moreover, it has been suggested that such a mechanism might not be important in other conditions such as abdominal myofascial pain syndrome.

\section{Strengths and limitations of the study}

The number of patients studied here was small, but the inclusion criteria were very strict; thus we believe that the data can be generalized with confidence. We recognize, however, that an issue that might be questioned is the interference of analgesic drugs, most commonly NSAIDs (35). Although all women claimed that they had not taken painkillers within the previous 3 or 4 days, this could not be verified because they tended to deny this self-medication behavior. In any case, the chronic anti-inflammatory action of NSAIDs may be due not only to inhibition of prostaglandin synthesis but also through activation of arginine-NO-cGMP pathways (36), inhibition of inducible nitric oxide synthase gene expression, and NO synthesis (37). Thus, we would have expected to detect lower NO levels in women with CPP before treatment, which was not the case. Further, it is known that neuronal nitric oxide synthase (nNOS) is the most important enzyme in the spinal cord during both development and maintenance of neuropathic and inflammatory pain $(38,39)$. However, our study does not allow for determining or making inferences about the origin of $\mathrm{NO}$ identified peripherally.

\section{Implications for future research or clinical practice}

NO may represent an objective systemic marker of reduced disease activity of endometriosis after treatment, and perhaps of reduced central sensitization. Additionally, the knowledge that inhibitors of nitric oxide synthase may act as analgesic agents (40) opens an opportunity for their use as a therapeutic target for this condition.

The present study does not permit us to make any conclusions about the source of NO. It may be locally produced in large amounts, and may reach the bloodstream after washout from the interstitial space in the affected region. The reasons are not entirely clear but it is known that persistent activation of nociceptors results in activation of the three known NO synthases, particularly inducible and neuronal nitric oxide synthase, hence increasing $\mathrm{NO}$ synthesis (14). On the other hand, we cannot explain why this would not occur in women with abdominal myofascial pain syndrome, and it is possible that, in women with CPP, more NO is produced by the vascular system in response to the prolonged and noncontinuous use of analgesic and/or anti-inflammatory drugs (about $80 \%$ of our patients selfmedicate with these substances more than twice a week) (3). Under these conditions, more NO would be produced in endothelial cells to compensate for the inhibitory effect of these analgesics. We consider this last hypothesis to be less probable because, assuming the same rates of self-medication, no difference would be expected between groups.

In summary, NO levels were elevated in women with CPP secondary to endometriosis, but not in women with CPP secondary to abdominal myofascial pain syndrome. Surgical treatment of endometriosis was associated with 
reduction in NO levels, which was correlated with a reduction in pain intensity and in distant pain thresholds. It is possible that $\mathrm{NO}$ is involved in the pathophysiology of pain secondary to endometriosis, possibly associated with central sensitization. In our opinion, this correlation supports NO as a potential marker in patients with CPP and

\section{References}

1. Latthe $\mathrm{P}$, Latthe M, Say L, Gulmezoglu M, Khan KS. WHO systematic review of prevalence of chronic pelvic pain: a neglected reproductive health morbidity. BMC Public Health 2006; 6: 177, doi: 10.1186/1471-2458-6-177.

2. ACOG Practice Bulletin No. 51. Chronic pelvic pain. Obstet Gynecol 2004; 103: 589-605.

3. Silva GP, Nascimento AL, Michelazzo D, Alves Junior FF, Rocha MG, Silva JC, et al. High prevalence of chronic pelvic pain in women in Ribeirão Preto, Brazil and direct association with abdominal surgery. Clinics 2011; 66: 1307-1312.

4. Fauconnier A, Chapron C. Endometriosis and pelvic pain: epidemiological evidence of the relationship and implications. Hum Reprod Update 2005; 11: 595-606, doi: 10.1093/ humupd/dmi029.

5. Kajihara H, Yamada Y, Kanayama S, Furukawa N, Noguchi T, Haruta $S$, et al. New insights into the pathophysiology of endometriosis: from chronic inflammation to danger signal. Gynecol Endocrinol 2011; 27: 73-79, doi: 10.3109/09513590. 2010.507292.

6. Shah JP, Danoff JV, Desai MJ, Parikh S, Nakamura LY, Phillips TM, et al. Biochemicals associated with pain and inflammation are elevated in sites near to and remote from active myofascial trigger points. Arch Phys Med Rehabil 2008; 89: 16-23, doi: 10.1016/j.apmr.2007.10.018.

7. Wu MY, Chao KH, Yang JH, Lee TH, Yang YS, Ho HN. Nitric oxide synthesis is increased in the endometrial tissue of women with endometriosis. Hum Reprod 2003; 18: 26682671, doi: 10.1093/humrep/deg484.

8. Korhonen R, Lahti A, Kankaanranta H, Moilanen E. Nitric oxide production and signaling in inflammation. Curr Drug Targets Inflamm Allergy 2005; 4: 471-479, doi: 10.2174/ 1568010054526359.

9. Cury Y, Picolo G, Gutierrez VP, Ferreira SH. Pain and analgesia: The dual effect of nitric oxide in the nociceptive system. Nitric Oxide 2011; 25: 243-254, doi: 10.1016/j.niox. 2011.06.004.

10. Meller ST, Cummings CP, Traub RJ, Gebhart GF. The role of nitric oxide in the development and maintenance of the hyperalgesia produced by intraplantar injection of carrageenan in the rat. Neuroscience 1994; 60: 367-374, doi: 10.1016/ 0306-4522(94)90250-X.

11. Wu J, Lin Q, McAdoo DJ, Willis WD. Nitric oxide contributes to central sensitization following intradermal injection of capsaicin. Neuroreport 1998; 9: 589-592, doi: 10.1097/00001756199803090-00005.

12. Schmidtko A, Tegeder I, Geisslinger G. No NO, no pain? The role of nitric oxide and cGMP in spinal pain processing. Trends Neurosci 2009; 32: 339-346, doi: 10.1016/j.tins.2009.01.010.

13. Holthusen $\mathrm{H}$, Arndt JO. Nitric oxide evokes pain in humans on intracutaneous injection. Neurosci Lett 1994; 165: 71-74, doi: 10.1016/0304-3940(94)90712-9. endometriosis, a characteristic that has not been reported previously.

\section{Acknowledgments}

Research supported by CNPq.
14. Koch A, Zacharowski K, Boehm O, Stevens M, Lipfert P, von Giesen $\mathrm{HJ}$, et al. Nitric oxide and pro-inflammatory cytokines correlate with pain intensity in chronic pain patients. Inflamm Res 2007; 56: 32-37, doi: 10.1007/s00011-007-6088-4.

15. Anbar M, Gratt BM. The possible role of nitric oxide in the physiopathology of pain associated with temporomandibular joint disorders. J Oral Maxillofac Surg 1998; 56: 872-882, doi: 10.1016/S0278-2391(98)90018-0.

16. Fan W, Huang F, Wu Z, Zhu X, Li D, He H. The role of nitric oxide in orofacial pain. Nitric Oxide 2012; 26: 32-37, doi: 10.1016/j.niox.2011.11.003

17. Hosseini A, Ehren I, Wiklund NP. Nitric oxide as an objective marker for evaluation of treatment response in patients with classic interstitial cystitis. J Urol 2004; 172: 2261-2265, doi: 10.1097/01.ju.0000144761.69398.be.

18. American Society for Reproductive Medicine. Revised American Society for Reproductive Medicine classification of endometriosis: 1996. Fertil Steril 1997; 67: 817-821, doi: 10.1016/S0015-0282(97)81391-X.

19. Simons DG, Travell JG, Simons LS, Cummings BD. Myofascial pain and dysfunction: the trigger point manual. 2nd edn. London: Lippincott Williams \& Wilkins; 1999.

20. Kamanli A, Kaya A, Ardicoglu O, Ozgocmen S, Zengin FO Bayik Y. Comparison of lidocaine injection, botulinum toxin injection, and dry needling to trigger points in myofascial pain syndrome. Rheumatol Int 2005; 25: 604-611, doi: 10.1007/ s00296-004-0485-6.

21. Melzack R. The McGill pain questionnaire: from description to measurement. Anesthesiology 2005; 103: 199-202, doi: 10.1097/00000542-200507000-00028.

22. Ruyssen-Witrand A, Tubach F, Ravaud P. Systematic review reveals heterogeneity in definition of a clinically relevant difference in pain. J Clin Epidemiol 2011; 64: 463-470, doi: 10.1016/j.jclinepi.2010.06.008.

23. Zigmond AS, Snaith RP. The hospital anxiety and depression scale. Acta Psychiatr Scand 1983; 67: 361-370, doi: 10.1111/ j.1600-0447.1983.tb09716.x

24. Botega NJ, Bio MR, Zomignani MA, Garcia C Jr, Pereira WA [Mood disorders among inpatients in ambulatory and validation of the anxiety and depression scale HAD]. Rev Saude Publica 1995; 29: 355-363, doi: 10.1590/S0034-89101995000 500004

25. Al-Azemi M, Refaat B, Amer S, Ola B, Chapman N, Ledger W. The expression of inducible nitric oxide synthase in the human fallopian tube during the menstrual cycle and in ectopic pregnancy. Fertil Steril 2010; 94: 833-840, doi: 10.1016/ j.fertnstert.2009.04.020

26. Giustarini D, Dalle-Donne I, Colombo R, Milzani A, Rossi R. Adaptation of the Griess reaction for detection of nitrite in human plasma. Free Radic Res 2004; 38: 1235-1240, doi: $10.1080 / 10715760400017327$ 
27. Ota H, Igarashi S, Hatazawa J, Tanaka T. Endothelial nitric oxide synthase in the endometrium during the menstrual cycle in patients with endometriosis and adenomyosis. Fertil Steril 1998; 69: 303-308, doi: 10.1016/S0015-0282(97)00478-0.

28. Khorram O, Lessey BA. Alterations in expression of endometrial endothelial nitric oxide synthase and alpha(v)beta(3) integrin in women with endometriosis. Fertil Steril 2002; 78: 860-864, doi: 10.1016/S0015-0282(02)03347-2.

29. Zervou S, Karteris E, Goumenou AG, Vatish M, Koumantakis EE, Hillhouse EW. The Glu298->Asp polymorphism of the endothelial nitric oxide synthase gene is associated with endometriosis. Fertil Steril 2003; 80: 1524-1525, doi: 10.1016/ S0015-0282(03)02204-0.

30. Tao F, Tao YX, Zhao C, Dore S, Liaw WJ, Raja SN, et al. Differential roles of neuronal and endothelial nitric oxide synthases during carrageenan-induced inflammatory hyperalgesia. Neuroscience 2004; 128: 421-430, doi: 10.1016/ j.neuroscience.2004.06.038.

31. Osborn BH, Haney AF, Misukonis MA, Weinberg JB. Inducible nitric oxide synthase expression by peritoneal macrophages in endometriosis-associated infertility. Fertil Steril 2002; 77: 46-51, doi: 10.1016/S0015-0282(01)02940-5.

32. Wiertelak EP, Furness LE, Watkins LR, Maier SF. Illnessinduced hyperalgesia is mediated by a spinal NMDA-nitric oxide cascade. Brain Res 1994; 664: 9-16, doi: 10.1016/ 0006-8993(94)91947-X.

33. Miclescu A, Gordh T. Nitric oxide and pain: 'Something old, something new'. Acta Anaesthesiol Scand 2009; 53: 11071120, doi: 10.1111/j.1399-6576.2009.02054.x.

34. Wu J, Fang L, Lin Q, Willis WD. Nitric oxide synthase in spinal cord central sensitization following intradermal injection of capsaicin. Pain 2001; 94: 47-58, doi: 10.1016/S0304-3959(01) 00340-2.

35. Sprott H, Gay RE, Michel BA, Gay S. Influence of ibuprofenarginine on serum levels of nitric oxide metabolites in patients with chronic low back pain--a single-blind, placebo controlled pilot trial (ISRCTN18723747). J Rheumatol 2006; 33: 25152518.

36. Tonussi CR, Ferreira SH. Mechanism of diclofenac analgesia: direct blockade of inflammatory sensitization. Eur J Pharmacol 1994; 251: 173-179, doi: 10.1016/0014-2999(94)90398-0.

37. Aeberhard EE, Henderson SA, Arabolos NS, Griscavage JM, Castro FE, Barrett CT, et al. Nonsteroidal anti-inflammatory drugs inhibit expression of the inducible nitric oxide synthase gene. Biochem Biophys Res Commun 1995; 208: 10531059, doi: 10.1006/bbrc.1995.1441.

38. Boettger MK, Uceyler N, Zelenka M, Schmitt A, Reif A, Chen $Y$, et al. Differences in inflammatory pain in nNOS-, iNOSand eNOS-deficient mice. Eur J Pain 2007; 11: 810-818, doi: 10.1016/j.ejpain.2006.12.008.

39. Guan Y, Yaster M, Raja SN, Tao YX. Genetic knockout and pharmacologic inhibition of neuronal nitric oxide synthase attenuate nerve injury-induced mechanical hypersensitivity in mice. Mol Pain 2007; 3: 29, doi: 10.1186/1744-8069-3-29.

40. De Alba J, Clayton NM, Collins SD, Colthup P, Chessell I, Knowles RG. GW274150, a novel and highly selective inhibitor of the inducible isoform of nitric oxide synthase (iNOS), shows analgesic effects in rat models of inflammatory and neuropathic pain. Pain 2006; 120: 170-181, doi: 10.1016/j.pain. 2005.10.028 